III Sección: Educación, producción y finanzas

\title{
Globalización, PyMEs y surgimiento de los Sistemas de Innovación Local
}

\author{
Carlos Alberto Hernández Medina \\ Centro Universitario Municipal Camajuaní. Villa Clara. Cuba \\ cahm862@uclv.edu.cu \\ https://orcid.org/0000-0003-3446-3014 \\ Alexander Báez Hernández \\ Universidad Central de Ecuador. Quito, Ecuador \\ albaez@uce.edu.ec \\ https://orcid.org/0000-0003-0585-1448 \\ Magdalys Alibet Carrasco Fuentes \\ Centro Universitario Municipal Camajuaní. Villa Clara. Cuba \\ alibet7305@uclv.edu.cu \\ https://orcid.org/0000-0002-0577-0712
}

Recibido: 20 de junio de 2020

Aceptado: 1 de noviembre de 2020

Resumen: Se analiza la influencia del proceso de Globalización sobre el surgimiento de nuevos paradigmas de desarrollo a nivel global, regional, nacional y local. Los cambios globales han generado nuevas formas de gestionar el conocimiento, han variado las relaciones de producción, comercialización y han permitido el surgimiento de nuevas interrelaciones en el entramado de la vida social. La gestión del conocimiento y la capacitación se convierten en preocupaciones fundamentales en todos los niveles de la sociedad. Estos nuevos comportamientos en la actividad productiva y de servicios obligaron a la creación de sistemas de innovación local para enfrentar los retos de la nueva situación global a nivel de territorio.

\section{() $(\Theta \odot$}

La Revista Estudios es editada por la Universidad de Costa Rica y se distribuye bajo una Licencia Creative Commons Atribución-NoComercial-CompartirIgual 3.0 Costa Rica. Para más información envíe un mensaje a 
Palabras claves: Globalización; desarrollo; gestión del conocimiento; territorio; capacitación; sistemas de innovación local.

\title{
Globalization, PyMEs and the emergence of Local Innovation Systems
}

\begin{abstract}
The influence of Globalization process on emergence of new development paradigms at global, regional, national and local level is analyzed. Global changes have generated new forms of knowledge management, the production and commercialization relationships have varied, and new interrelations in the lattice of the social life arose. Knowledge management and training become fundamental occupations at all society levels. These new behaviours in productive and services activity forced to the creation of Local Innovation Systems to front the challenges from the new global situation to territory level.
\end{abstract}

Keywords: Globalization; development; knowledge management; territory; training; Local Innovation Systems.

Patria es humanidad, es aquella porción de la humanidad que vemos más cerca, y en que nos tocó nacer. José Martí, (1893).

\section{Globalización y Nuevas Tecnologías, medios para el cambio de paradigma.}

En los últimos años se han producido grandes e importantes transformaciones en el escenario socioeconómico internacional. La globalización de los mercados, la introducción gradual de las Nuevas Tecnologías de la Información y de la Comunicación (NTICs) en el escenario productivo, social internacional y la emergencia de nuevos paradigmas técnico-organizacionales en el ámbito empresarial son la cara visible de estos cambios globales. (Báez et al., 2018)

El contexto anterior se caracteriza por: volatilidad de la demanda y segmentación de los mercados, posibilidad de combinar escala y gama, acortamiento del ciclo de

\section{(C) $(\Theta \odot \odot$}

La Revista Estudios es editada por la Universidad de Costa Rica y se distribuye bajo una Licencia Creative Commons Atribución-NoComercial-CompartirIgual 3.0 Costa Rica. Para más información envíe un mensaje a 
vida de los productos e incertidumbres estratégicas asociadas al nuevo escenario mundial. Dentro de ese escenario, las empresas y agentes económicos han debido enfrentar una creciente presión competitiva surgida como consecuencia de este conjunto de cambios. Se ha renovado el concepto de competitividad, que ahora se percibe como un fenómeno de naturaleza solamente macroeconómica y sectorial determinada por ventajas comparadas estáticas o por la dotación factorial. Con la globalización la posibilidad de competir depende del desarrollo de producciones de alto valor agregado y alta calidad.

Para muchos teóricos la globalización aún no tiene definición precisa. Pero una definición general que podría ajustarse al término sería que_ “...abarca todo, desde los mercados financieros hasta Internet, pero ofrece muy poca condición de la comprensión humana contemporánea." (Held, 2002)

El nuevo escenario globalizado hace cambios en los factores que condicionan la competitividad de los bloques económicos, países, regiones, ámbitos locales, empresas y en las formas en que se da la competencia. El nuevo escenario competitivo global, intensivo en Tecnologías de la Información y la Comunicación genera oportunidades, pero el cambio en los factores que deciden la ventaja competitiva plantea nuevos desafíos y amenazas. (Kotler, 2000)

La incertidumbre se ha convertido en una característica ineludible del escenario competitivo y con ella se enfrentan las elecciones y decisiones productivas y de servicios. Según Boscherini y Yoguel (1996), en el marco del proceso de competencia y búsqueda de diferenciación impulsada por la globalización, las organizaciones buscan incrementar la capacidad innovativa. Esta se define como la potencialidad de transformar conocimientos genéricos en específicos a partir de las competencias derivadas del aprendizaje formal e informal, para diseñar e implementar estrategias ajustadas al nuevo escenario globalizado, que permitan lograr y mantener la ventaja competitiva.

\section{(c) (i) (2) (2)}

La Revista Estudios es editada por la Universidad de Costa Rica y se distribuye bajo una Licencia Creative Commons Atribución-NoComercial-CompartirIgual 3.0 Costa Rica. Para más información envíe un mensaje a 
Las Tecnologías de la Información y la Comunicación cambian los procesos de generación y difusión de innovaciones que necesitan una gran capacidad de adaptación en dos aspectos: 1) calificación de los actores productivos, motivación e implicación con los objetivos de la empresa y 2) la mejora productiva que ocurre en la globalización a partir de la difusión de innovaciones tecnológicas, acceso al conocimiento tecnológico, cooperación en investigación y desarrollo para proyectos de innovación y adquisición de nueva tecnología externa. Estos son los hechos más destacados en lo referente a los lineamientos del nuevo paradigma tecnológico. La globalización se vincula también al incremento del intercambio de bienes, servicios e información que promueve una interdependencia cada vez mayor entre las diferentes partes del mundo. Por eso, las empresas amplían su campo de acción y agregan a sus funciones tradicionales el diseño e implementación de innovación tecnológica que tiende a generar ventajas competitivas en diseño y productividad de sus bienes (Báez et al. 2017). Para afrontar este desafío, algunas empresas presentan como fortaleza una fluida relación con su entorno que les permite conocer con mayor certeza la realidad económica, social y político-institucional sobre la que tienen que actuar.

Actualmente el desafío que significa implementar estrategias en el seno de la globalización imperante se transforma en una tarea nada fácil para aquellos empresarios que desean innovar con tecnología su empresa. (Kotler, 2000).

\footnotetext{
(c) (i) (2) (2)

La Revista Estudios es editada por la Universidad de Costa Rica y se distribuye bajo una Licencia Creative Commons Atribución-NoComercial-CompartirIgual 3.0 Costa Rica. Para más información envíe un mensaje a revistaestudios.eeg@ucr.ac.cr.
} 


\section{La Globalización y los cambios en la producción.}

Las transformaciones antes mencionadas han impactado en la organización social del trabajo en el ámbito de la empresa, los espacios nacionales, internacionales y las condiciones de valorización del capital. Ante esa situación cabe preguntarse cómo se situará lo local frente a las tendencias que imperan en el ámbito global. En ese sentido, Arocena (1998) asegura que hay tres maneras de situarse en torno a la relación entre lo global y lo local. La primera sería afirmar el carácter determinante de lo global sobre lo local, en esta posición, se establece que las macro-tendencias se reproducen en los espacios locales y que resulta casi imposible un planteo de desarrollo endógeno. La segunda premisa postula lo local como alternativa a los males de la globalización (en esta perspectiva se apela a la generación de conductas de reactivación en el ámbito local para resistir a lo global). Finalmente, la tercera destaca la articulación entre lo local y lo global (en ese enfoque se promueve una comprensión compleja de la sociedad contemporánea para superar las visiones anteriores). El autor se identifica con la tercera posición y afirma que la globalización no sólo es una amenaza, sino también una oportunidad para desplegar el potencial de lo local ante las condiciones emergentes a escala global. Se trata de propiciar una interacción permanente entre actor y sistema. Para finalizar con relación a la globalización y el desarrollo empresarial, autores como Brusco (1992) y Piore (1992) apoyan la articulación entre la estructura productiva y la lógica global. Consideran que la globalización impone un nuevo modelo de producción denominada flexible o post-fordista, basado en estrategias de innovación tecnológica y organización empresarial. Por tanto, los espacios locales no pueden quedar al margen de dicha realidad y están desafiados a estimular la capacidad de competencia de sus agentes. La innovación no es una actividad aislada ya que las empresas entablan relaciones entre ellas. Es un proceso acumulativo, no sólo porque se asienta en conocimientos ya desarrollados, que

La Revista Estudios es editada por la Universidad de Costa Rica y se distribuye bajo una Licencia Creative Commons Atribución-NoComercial-CompartirIgual 3.0 Costa Rica. Para más información envíe un mensaje a revistaestudios.eeg@ucr.ac.cr. 
serán transformados y combinados, sino porque se introducen en la economía por etapas.

\section{Cambios productivos que han ocurrido en las fronteras nacionales}

En lo que se refiere a la estructura interna la capacidad endógena de los actores, el grado de desarrollo del ambiente en el que actúan y la pertenencia a una red o trama de actores se han convertido en elementos claves para la creación de ventajas competitivas. Para comprender el significado del cambio es importante tener en cuenta la transformación que realizan las empresas en su proceso de modernización. Ruggie (1993) señaló que esta etapa marca una nueva frontera de práctica óptima tecnológica y organizativa. Esta está encarnada en tecnologías genéricas aplicables en todo el aparato productivo, producto o servicio, tipo de organizaciones y actividades. Su gran potencial de generación de riqueza consiste en esto.

Por un lado, está el surgimiento de industrias nuevas que ofrecen oportunidades inéditas de innovación e inversión, un nuevo patrón tecnológico y organizativo y herramientas para modernizar todo el resto de la economía, para así llevarla a un plano de productividad y efectividad superior. Esta fuerza innovadora y renovadora abre una ventana de oportunidad para las empresas y países que logren adoptar el nuevo paradigma. Por otro lado, todo el aparato productivo basado en el paradigma anterior queda obsoleto y debe ser modernizado o corre el riesgo de ser desplazado del mercado (Ferrer, 1996).

Al emerger nuevas formas productivas, más intensivas en información, se produjeron cambios en la organización de la producción. Esto induce a las organizaciones a acelerar el papel de los procesos innovativos formales e informales en busca de competitividad. Ello se debe al aumento de las

La Revista Estudios es editada por la Universidad de Costa Rica y se distribuye bajo una Licencia Creative Commons Atribución-NoComercial-CompartirIgual 3.0 Costa Rica. Para más información envíe un mensaje a revistaestudios.eeg@ucr.ac.cr. 
incertidumbres estratégicas, segmentación de la demanda, volatilidad de los mercados, cambios en el concepto de eficiencia y la posibilidad de combinar economías de escala. La importancia de ciertos factores va en aumento, tal es el caso de la competitividad, calidad, servicios de venta, adaptación al cliente y capacidad de diseño.

La segmentación de los mercados y el acortamiento del ciclo de vida de los productos replantea el proceso innovativo* e involucra nuevos agentes y modalidades operativas. Así, las actividades innovatidoras implican acciones que incluyen labores orientadas al desarrollo de la calidad, las mejoras y desarrollos acumulativos de tipo incremental. Con el transcurso del tiempo estos cambios son incorporados a los productos, procesos productivos, modalidades de organización y a las formas de comercialización. Todo esto promueve también una renovación de las instituciones gubernamentales encargadas del desarrollo tecnológico.

\section{Surgimiento de los Sistemas Nacionales de Innovación.}

Con el surgimiento de los Sistemas Nacionales de Innovación (SNI) pueden identificarse dos dimensiones. En un primer nivel, está la estructura de la producción en la que ocurren los procesos de interacción y aprendizaje de tipo acumulativo. En un segundo nivel, se identifica a la organización institucional o conjunto de rutinas, normas, reglas y leyes que rigen el comportamiento de los agentes. Además, determinan la forma en que se realiza el aprendizaje interactivo, el ritmo y la dirección de la innovación. Por eso, la gestión del conocimiento se convierte en preocupación fundamental para los actores del sistema. Las empresas no solo organizan cursos de capacitación a todos los niveles, algunas inclusive han creado sus propias universidades. En este escenario es necesario invertir en la investigación para el futuro y dirigir la tecnología hacia el mejoramiento directo e

\section{(c) (i) (2) (-)}

La Revista Estudios es editada por la Universidad de Costa Rica y se distribuye bajo una Licencia Creative Commons Atribución-NoComercial-CompartirIgual 3.0 Costa Rica. Para más información envíe un mensaje a revistaestudios.eeg@ucr.ac.cr. 
inmediato de las redes de producción y la calidad de la vida desde un sistema de ciencia y tecnología impulsado por la oferta a una red interactiva con los productores. Se justifica la creación del Sistema Nacional de Innovación definido por Freeman (2003) como red de instituciones del sector público y privado cuyas actividades e interacciones inician, importan, modifican y difunden nuevas tecnologías.

Esto parte del supuesto de que el Sistema Nacional de Innovación es una creación social y no gubernamental. Incluye el entorno en que se estimula y apoya la innovación; la calidad de los vínculos entre proveedores, productores y usuarios; el régimen de educación y capacitación; y las organizaciones públicas y privadas que facilitan el cambio técnico; las leyes, los reglamentos e incluso las ideas y actitudes hacia la tecnología y el cambio.

\section{Influencia y cambios a nivel local.}

En este nuevo escenario se destacan de manera exponencial las respuestas de los agentes que apuntan a diferenciar productos, desarrollar mejoras incrementales en productos, procesos y en la organización, buscar nuevas formas de vinculación con el mercado y lograr un creciente aumento de la calidad. En la búsqueda de diferenciación, implícita en el proceso de competitividad, los agentes apuntan a aumentar lo que denominaremos capacidad innovativa.

Este concepto engloba la potencialidad de los actores para transformar conocimientos genéricos en específicos a partir de sus competencias y su acumulación dinámica que involucra aprendizajes formales e informales, de tipo codificado y tácito (Ernest et al., 1995; Lall, 1992). Estas competencias, tanto de stock como de flujo pueden definirse como el conjunto de conocimientos, rutinas y

\section{(c) (i) (2)}

La Revista Estudios es editada por la Universidad de Costa Rica y se distribuye bajo una Licencia Creative Commons Atribución-NoComercial-CompartirIgual 3.0 Costa Rica. Para más información envíe un mensaje a 
habilidades tecnológicas y organizativas, formales e informales que las organizaciones generan para llevar a cabo los desarrollos mencionados.

Debido al carácter sistémico de la competitividad y la naturaleza interactiva de la innovación, esta se concibe como un proceso de aprendizaje en que se introducen nuevos conocimientos o se combinan otros existentes para generar nuevas competencias. Esto produce una resignificación de la función del ambiente local y sus instituciones en el desarrollo de las capacidades innovativas de las organizaciones y empresas. (Lavagna, 1999).

Desde esta perspectiva, el ambiente local se entiende como el conjunto de instituciones, agentes locales y sus interrelaciones. Es un espacio público que, en el extremo positivo, puede dar lugar a procesos de eficiencia colectiva definidos como ventaja competitiva derivada de economías externas y de la acción conjunta de los agentes. De este modo, la existencia de un ambiente favorable se manifiesta en las acciones de los agentes que componen la sociedad civil, que a partir de la cooperación, competencia y presión mutua generan una tensión colectiva que favorece el desarrollo de estrategias innovadoras.

En este tipo de ambientes se genera un capital social construido a partir de vinculaciones complejas que derivan en desarrollo de confianza que facilita la reducción de incertidumbres y circulación de conocimiento codificado y tácito (Putnam, 1993). Aunque un ambiente sinérgico tiende a lograr una tendencia proactiva, las conductas de los agentes y el aprovechamiento de las externalidades generadas requieren de umbrales mínimos de competencias que pocos agentes locales poseen. Estas no se pueden adquirir sin un proceso evolutivo de creación y destrucción de rutinas y convenciones que regían los procesos hasta el presente (Gregersen y Johnson, 1996). Por ello, a diferencia de otras experiencias, el ambiente local suele tener una escasa influencia en el proceso de formación de

La Revista Estudios es editada por la Universidad de Costa Rica y se distribuye bajo una Licencia Creative Commons Atribución-NoComercial-CompartirIgual 3.0 Costa Rica. Para más información envíe un mensaje a 
ISSN 1659-3316

Hernández Medina Carlos Alberto

Báez Hernández Alexander

Carrasco Fuentes Magdalys Alibet

competencias de las empresas. En el contexto de las nuevas condiciones de producción y mercado, los procesos innovadores pasan de ser un fenómeno de carácter individual, y a menudo de naturaleza incremental, a otro que se incluye en el plano colectivo. En él resulta fundamental tanto la capacidad de interacción y cooperación como la presencia de una estructura institucional adecuada capaz de promover las actividades innovativas de los agentes económicos. La capacidad individual de los agentes es una condición necesaria que está aún más desarrollada en ambientes sinérgicos. Las innovaciones no son un grupo de eventos aislados y están inevitablemente vinculadas con otros, tanto en su basamento técnico y científico, como en sus conexiones físicas con otras partes del sistema económico. Para Rosenberg y Rolt (1989), en la primera Revolución Industrial, la tecnología de las máquinas-herramienta tuvo una influencia decisiva sobre toda la innovación en materia de bienes de capital que se desarrolló durante los siglos XVIII y XIX. En otro sentido, los procesos informales de aprendizaje e interacción en el interior de la empresa comienzan a ser considerados fuentes relevantes en el desarrollo de las actividades innovativas realizadas por ejemplo, en las pequeñas y medianas empresas (PyMES).

El creciente desarrollo de nuevos mercados y nuevas tecnologías desde comienzos de la década de 1980, condicionan y amenazan a las industrias locales. La creciente transformación y segmentación de los mercados implica una competencia internacional más intensa que, acentuada con la volatilidad de los productos, exige una innovación sistemática y un uso mayor de servicios especializados, muchos de los cuales sólo pueden presentarse en las grandes ciudades.

La emergencia de nuevas potencias industriales y económicas que, gracias a la combinación de sus especiales estructuras sociales con la utilización masiva de las nuevas tecnologías de fabricación flexible, han logrado ventajas casi inalcanzables y presentan una realidad casi insoslayable para la sociedad. La mejora de los

La Revista Estudios es editada por la Universidad de Costa Rica y se distribuye bajo una Licencia Creative Commons Atribución-NoComercial-CompartirIgual 3.0 Costa Rica. Para más información envíe un mensaje a 
niveles educativos y la creciente influencia de los medios de comunicación están transformando rápidamente las condiciones y percepciones de los trabajadores. Finalmente, la nueva revolución tecnológica, forma parte de un proceso de cambio de paradigma que ha aportado nuevas pautas cuyo resultante es un nuevo modo de producción y organización de la economía.

No obstante, lo que más dificulta y retarda la transición del viejo al nuevo paradigma es la necesidad de la adopción masiva del más reciente modelo tecnológico y organizativo, de un nuevo sentido común, de otra forma de pensar la eficiencia y la búsqueda de la adaptabilidad

\section{Innovación tecnológica como motor del crecimiento}

La crisis del modelo industrial de posguerra, el estancamiento de la demanda junto con la globalización y la emergencia de nuevos paradigmas intensivos en información han producido importantes transformaciones en la organización de la producción. Esto ha obligado a las empresas a aumentar el rol asignado a los procesos innovativos, formales e informales en la búsqueda de la competitividad.

La innovación tecnológica ha tenido como eje central establecer que los procesos de producción, difusión y adopción de nuevas tecnologías mejoradas deben ser integrados de manera totalmente central en la teoría de la acumulación del mercado y de la competencia capitalista. (Manzanares, 1996: 16-26).

Si se toma como ejemplo el sector industrial se observa que hay únicamente gasto en investigación y desarrollo porque la rentabilidad del capital y la competitividad de las empresas dependen de la innovación, así como de la capacidad para comprender sus posibilidades. Schumpeter (1994) estableció que el motor del desarrollo capitalista a largo plazo reside en este proceso de innovación y, al mismo tiempo, propuso una teoría sobre los ciclos de negocios que se centraban en la forma en que la economía capitalista las absorbe y digiere.

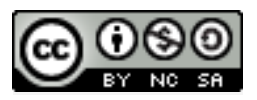

La Revista Estudios es editada por la Universidad de Costa Rica y se distribuye bajo una Licencia Creative Commons Atribución-NoComercial-CompartirIgual 3.0 Costa Rica. Para más información envíe un mensaje a revistaestudios.eeg@ucr.ac.cr. 
En su modelo los cambios tecnológicos exógenos estimulan los gastos de inversión, cuyas variaciones, generan al mismo tiempo inestabilidad cíclica. La innovación no se reduce sólo a las actividades aisladas orientadas a desarrollar nuevos productos y procesos, sino que también involucra el conjunto de desarrollos y mejoras graduales realizadas en las distintas áreas como son organización, comercialización y producción y las actividades destinadas al desarrollo de la calidad (Manzanares, 1996). El desarrollo de las innovaciones también se genera a partir de distintas actividades rutinarias efectuadas en la empresa que no necesariamente están vinculadas con el área productiva. La interacción del personal de la empresa, el intercambio continuo de opiniones para resolver problemas o para enfrentar nuevas situaciones, las respuestas que surgen y que se utilizan para que la empresa funcione y mejore su eficiencia económica constituyen una fuente relevante de inputs para el desarrollo de actividades innovativas. Por ello, aumentan los espacios de las actividades innovativas y su difusión en la estructura empresarial. Las actividades innovativas tienen un impacto en el conjunto de áreas operativas de la organización, lo que genera una retroalimentación continua entre su sendero madurativo y los resultados que producen. Así, la búsqueda continua de técnicas eficientes de gestión orientadas a la mejora y a la racionalización del funcionamiento de las distintas áreas de la empresa llevan a la implementación del concepto de calidad.

Azzone y Cainarca (1993) plantean la diferencia entre calidad interna que se da a nivel proceso y de la calidad externa que se da a nivel producto. La primera es la habilidad para minimizar las tareas que no generan valor agregado (desperdicio, tiempo de espera, colas, entre otros). La segunda apunta a que los productos se adapten a las especificaciones de diseño y satisfagan a los consumidores en términos de seguridad y prestaciones.

\section{(C) $(\Theta \odot$}

La Revista Estudios es editada por la Universidad de Costa Rica y se distribuye bajo una Licencia Creative Commons Atribución-NoComercial-CompartirIgual 3.0 Costa Rica. Para más información envíe un mensaje a 
En la estructura organizativa de la empresa, el desarrollo de innovaciones y la forma en que se aprovechan sus resultados constituye un patrimonio específico de tipo dinámico, fuertemente influido por la capacidad de gestión y el planteamiento estratégico

\section{El proceso innovativo y la creación de capacidades en la organización.}

El desarrollo de actividades innovativas constituye una condición necesaria pero no suficiente para garantizar un buen comportamiento económico. Las elecciones estratégicas realizadas por la administración deben guardar una estrecha relación con los avances innovativos de la empresa. Es necesario verificar un continuo y sincrónico intercambio entre el proceso estratégico de decisión y el proceso de innovación. Desde esta perspectiva consideramos que existen fuertes interacciones y vínculos entre el proceso de decisión y las actividades de mejora. Eso significa que este tipo de actividades constituyen una premisa para cualquier decisión estratégica vinculada con la gestión de la empresa que tienen impacto y consecuencias sobre el conjunto de actividades desarrolladas en ella. Sin embargo, el aprovechamiento integral de los resultados de las actividades innovativas depende básicamente de las capacidades de la empresa para elaborar y llevar a cabo estrategias competitivas coherentes. En el proceso de innovación confluyen distintos conocimientos y capacidades que están presentes en las diferentes áreas de la empresa cuyo aprovechamiento depende de la cultura organizacional. A lo largo del tiempo la interacción entre este conjunto de factores va generando un patrimonio de competencias, en muchos casos intangibles y específicas a la empresa, que determinan y condicionan su capacidad innovativa (Dierickx et al, 1990).

\section{(C) $(\Theta \odot$}

La Revista Estudios es editada por la Universidad de Costa Rica y se distribuye bajo una Licencia Creative Commons Atribución-NoComercial-CompartirIgual 3.0 Costa Rica. Para más información envíe un mensaje a 
Las características y las capacidades de los recursos humanos que trabajan en la empresa son de fundamental relevancia en este proceso. Por lo tanto, los rasgos específicos de la gestión y del estilo de conducción constituyen elementos centrales para la formación de competencias*. La producción y el desarrollo de conocimientos en el interior de la organización constituye un proceso dinámico, continuo y acumulativo que modifica y crea las habilidades organizacionales y tecnológicas. En este proceso el aprendizaje individual y colectivo juega un rol central y determina que las competencias sean recursos dinámicos moldeables de acuerdo con la visión estratégica de la empresa.

La capacidad innovativa de las empresas se puede definir como la potencialidad de idear, planificar y realizar mejoras a partir del uso de los conocimientos tecnológicos y organizativos formales e informales para cubrir sus necesidades específicas. Lo anterior va a permitir que se aprovechen las competencias desarrolladas a lo largo del desarrollo de la empresa. También la capacidad innovativa puede asociarse con lo que Lall (1992) denomina technological capabilities para explicar la diferente capacidad de las empresas para utilizar, incorporar y modificar la tecnología. Por su parte, Ernest et al. (1995), Mitelka (1993), Katz (1987) y Pietrobelli (1995) consideran que la innovación es un proceso mediante el cual las empresas aprenden y desarrollan bienes y servicios nuevos para ellos, aunque no necesariamente nuevos para los competidores. Sin embargo, la actividad innovativa se desarrolla en un ambiente económico con diversos tipos de incertidumbres, que son decodificadas de distinta manera por las empresas en función de su cultura organizacional y el grado de desarrollo de sus competencias. Estas competencias acumuladas permiten decodificar de forma distinta el conjunto de informaciones y señales del ambiente en términos de conocimientos específicos vinculados con sus particularidades. Este conjunto de códigos, que son desarrollados en forma desigual por las empresas y que, por lo tanto, asumen rasgos específicos, condicionan la calificación de los recursos humanos presentes 
en la empresa y por tanto sus competencias y el grado de complejidad de las innovaciones realizadas.

El proceso innovativo en las empresas adquiere un carácter multidimensional lo que permite diferenciar dos planos en el mismo. En primer lugar, destaca el conjunto de elementos ubicados a nivel micro y en segundo lugar el ambiente, es decir el entorno socio institucional y su influencia en el proceso de construcción de competencias. Entre los elementos ubicados a nivel micro que influyen sobre el proceso innovativo a partir de formación de competencias deben señalarse principalmente las modalidades de gestión, las características personales y educativas y la capacidad emprendedora de los empresarios, la historia previa de las empresas en términos de acumulación de activos competitivos tangibles e intangibles, los procesos de aprendizaje y la capacidad de diseño de estrategias. Estos elementos se manifiestan en cada empresa mediante diferentes rutinas, normas y reglas, desarrolladas a lo largo de su sendero madurativo que influyen sobre la capacidad de acumular competencias y que condicionan, a su vez, las modalidades bajo las que se manifiesta el proceso de aprendizaje junto con las capacidades de interacción con otros agentes. El ambiente constituido por el conjunto de instituciones, agentes y relaciones existentes entre ellos, influye de manera decisiva en el grado de desarrollo de actividades innovativas. Por tanto, el proceso de innovación en ellas es el resultado de la interacción dinámica de las competencias desarrolladas a lo largo del tiempo, el aprendizaje obtenido con la experiencia y la cultura organizacional.

La empresa moderna está adoptando un sistema de producción flexible capaz de fabricar una gama de productos cambiantes que se adapten a las variaciones de la demanda en cantidad y calidad. Por esta razón, perseguir solamente economías de escala es ahora menos rentable que lograr economías de especialización y de gama. (Thurow, 1996) Otro de los grandes lineamientos tiende a la superación del modelo* de producción intensivo en el uso de energía y materias primas. Este 
patrón, que por décadas sustentó el crecimiento de la sociedad de consumo y luego condujo a la crisis energética y ecológica, está siendo sustituido por un método alternativo de producción intensiva en información, conocimientos y servicios. Este es capaz de permitir, al menos desde el terreno tecnológico, una redefinición de los modos de vida y de brindar formas de manejar eficazmente las cuestiones ambientales. Bajo las nuevas condiciones, crece la proporción intangible en el perfil de producción, así como crecen la innovación y los servicios en el valor agregado de cada producto.

Localidades vs, contexto nacional e internacional. Desafíos y oportunidades.

En los principales distritos industriales de los países europeos se puede observar que las pequeñas y medianas empresas buscan constantemente estrategias, formas de organización y producción más flexibles y novedosas con el objetivo de responder a los crecientes desafíos de la globalización y las cambiantes exigencias del mercado. Para alcanzar este objetivo no agotan sus instancias sólo con incorporar tecnologías modernas, sino que simultáneamente adoptan nuevas formas de organización de los procesos de investigación, diseño, gestión, producción y comercialización, entre otros. Este amplio esquema definido como un proceso de transformaciones no resulta ser nada sencillo. Por el contrario, es muy complejo y asume características específicas según los sectores, regiones y países, a su vez, involucra interacciones que al complementarse permiten, en la mayoría de los casos, excelentes resultados.

En la actualidad se observa que la posibilidad de acceder a niveles crecientes de competitividad y mantenerlos a largo plazo, no puede circunscribirse a la acción de unos pocos empresarios locales. Todo esto en la actualidad es un común denominador dentro del tejido industrial de las PyMEs locales. Es importante mencionar también que deben pensarse en términos similares las reglas de juego

La Revista Estudios es editada por la Universidad de Costa Rica y se distribuye bajo una Licencia Creative Commons Atribución-NoComercial-CompartirIgual 3.0 Costa Rica. Para más información envíe un mensaje a revistaestudios.eeg@ucr.ac.cr. 
del desarrollo local que se pretende diagramar a futuro para poder aprovechar el entorno local y las variables económicas que resultan favorables en términos generales.

Al retomar experiencias internacionales es posible observar que los casos exitosos son explicados a partir de un conjunto de variables que muestran con claridad que el funcionamiento local en su conjunto es el que permite lograr una base sólida para el desarrollo de la competitividad, tanto interna como externa. Sería importante que las pequeñas y medianas empresas comenzaran a reemplazar los esfuerzos individuales en pos de un mejor lineamiento competitivo. Este objetivo debe estar acompañado por innumerables aspectos que conforman el entorno local desde la infraestructura física y tecnológica que posee hasta su organización interna. Combinar todo lo anterior para funcionar como una organización, con interacción constante entre sus miembros, flujos efectivos de información y decisiones, y una sinergia, que es más que la suma de las habilidades y conocimientos individuales, es considerado en la actualidad como el principal mecanismo a la hora de obtener resultados satisfactorios por parte de las pequeñas y mediana empresas.

Es conceptualmente útil considerar el desarrollo de la competitividad a nivel de las pequeñas y medianas empresas locales, como una inversión más, a las ya establecidas como patrón de modelo a seguir. Estas consideraciones también sirven para profundizar en la integración y en el desarrollo de la industria local, este último también debe estar definido por las PyMEs en su conjunto. Fuera de los beneficios productivos, estas interrelaciones aceleran la difusión de tecnologías, incrementan la especialización, aumentan la flexibilidad industrial y generan empleo dentro de la localidad.

En cuanto a los procesos de aprendizaje, se puede observar que no surgen automáticamente por el transcurso del tiempo. Son justamente el resultado positivo de los esfuerzos deliberados y explícitos orientados a la generación de acervos tecnológicos y a la capacitación en temas de recursos humanos. Esto significa 
desarrollar una estrategia tecnológica y productiva asociada a inversiones orientadas a la generación y adopción de cambios técnicos permanentes que induzcan una maduración que no es automática ni instantánea y requiere esfuerzos permanentes y conscientes por parte de los actores locales.

Haciendo un análisis desde un punto de vista microeconómico, dentro de la década pasada en lo que a pequeñas y medianas empresas respecta, se observa que hay mayor presencia de funciones de producción menos intensivas en trabajo, un abandono de la mayor parte de los esfuerzos tecnológicos locales en la generación de nuevos productos y procesos, una descentralización de las actividades basada en la sustitución de valor agregado local por abastecimiento externo y una reducción de la producción local.

\section{Conformación de estrategias de mediano plazo para las PyMEs.}

Al momento de sugerir una posible estrategia para las PyMEs locales, se pueden identificar tres objetivos generales que permitan un cambio más adecuado en la industrialización de las pequeñas y medianas empresas. Estos objetivos se plantean luego de observar y tener en cuenta la realidad que hoy presentan las empresas regionales. Su identificación está orientada a:

1. Promover un mejor ambiente de producción, eliminar alteraciones y obstáculos que condicionen* el desarrollo competitivo del mercado.

2. Impulsar un ambiente favorable para el aumento de la competitividad y la cooperación.

3. Disminuir la problemática de empleo calificado que presenta el mercado local.

Es importante sumar el desafío de aumentar la calidad del patrón de especialización productivo con la incorporación de un mejor aprovechamiento de los recursos naturales. Todo ello en conjunto puede generar una complementación que beneficie a cada uno dentro de sus actividades y al conjunto del territorio en 
general. La posibilidad de orientar la producción en forma clara y definida hacia la competitividad con productos diferenciados y con la gradual incorporación de mano de obra calificada, sumado a la alta absorción de tecnología, podría considerarse una alternativa que permitiría superar las dificultades que poseen las pequeñas y medianas empresas para lograr alta competitividad*. Deben desarrollar capacidades tecnoproductivas para mejorar la eficiencia. También, adaptarse para incorporar nuevas tecnologías en el proceso de producción. Deben rescatar los activos tecnoproductivos acumulados en su trayectoria evolutiva. Todo esto les permitirá superar más rápido los obstáculos que ese nuevo proceso genera. La competitividad de sus productos y su presencia en los mercados externos, son objetivos que las PyMEs deben replantearse como estrategia para implementar nuevos criterios en los procesos de inversión y de innovación tecnológica.

Potenciar el tramado de PyMEs en un nuevo escenario competitivo como el actual, el desarrollo de capacidades y la mayor integración de estas empresas al tejido productivo, a partir de su participación en redes de firmas, y de una activa vinculación con las grandes empresas, permitirá una mejor lectura de las señales del mercado. Lo anterior es de fundamental importancia para que las PyMEs desplieguen nuevas estrategias competitivas y generen desarrollos novedosos que posibiliten su inserción activa en el mercado internacional. De este modo se tenderá a aumentar el número de PyMEs con posibilidades de adaptarse a las nuevas reglas del juego, mediante el logro de una mayor especialización, el aumento de las escalas en las que operan y de la implementación de normas internacionales de calidad entre otras. Tender a un creciente equilibrio territorial a través del fortalecimiento de las articulaciones de las PyMEs con instituciones públicas y privadas locales permitirá generar sinergias y potenciar la capacidad de acumulación en el ámbito regional. Es importante destacar que operar a través del desarrollo de corredores comerciales adecuados desde el ámbito local pensando 
en el comercio regional, significaría reducir los elevados costos de transacción derivados de los movimientos que operan dentro de la región demandan.

\section{Referencias bibliográficas}

Arocena, J. (1998), El desarrollo local, un desafío contemporáneo. Caracas, Nueva Sociedad.

Báez, A.; Hernández, C; Perdomo, J.; Garcés, R., Carrasco, M, (2018). Modelo de gestión del conocimiento para el desarrollo agropecuario local. Estudios Sociales. 28(51), 2-26. DOI: https://dx.doi.org/10.24836/es.v28i51.517

Báez, A.; Hernández, C; Carrasco, M. (2017). Gestión universitaria del conocimiento para el desarrollo local. Lámpsakos. 12(18), 44-57. Julio - Dici. ISSN: 2145-4086. DOI: http://dx.doi.org/10.21501/21454086.2317

Báez, A.; Hernández, C; Perdomo, J.; Garcés, R., Carrasco, M. (2018). Praxis de una Red Local GUCID y Desarrollo Local. Rev. Ing. Industrial. 18(3). 253-266. ISSN 0717-9103.

Boscherini, F. y Yoguel, G. (1996). Algunas reflexiones sobre la medición de los procesos innovativos: La relevancia de los procesos informales e incrementales, Redes, 2(19). 12-26. Univ. de Quilmes.

Dierickx, C., Pralad, C y Hamel, N, (1990). La estrategia como objeto de estudio: ¿por qué buscar un nuevo paradigma? Harvard Deusto Business Review. 23(75). 48-55. Nov. - diciembre.

Ernest, C., Mitelka, N., Katz, P. y Pietrobelli, S. (1995). La capacidad innovativa y el fortalecimiento de la competitividad de las firmas: El caso de las PYMES exportadoras argentinas. CEPAL. Alianza Editorial.

Ferrer, A. (1996). Historia de la Globalización. FCE. México.

\section{(c) (i) (2)}

La Revista Estudios es editada por la Universidad de Costa Rica y se distribuye bajo una Licencia Creative Commons Atribución-NoComercial-Compartirlgual 3.0 Costa Rica. Para más información envíe un mensaje a revistaestudios.eeg@ucr.ac.cr. 
Freeman, C. (2003). Crisis estructurales de ajuste, ciclos económicos y comportamiento de la inversión. Ciencia, tecnología y crecimiento económico. Compilado - CEIL, PIETTE CONICET. Trabajo y Sociedad, 21/45). 211-243.

Gregersen, B, y Johnson, B. (1996). Learning economies, innovation systems and European Integration. Regional Studies. 31(51). 23-45.

Held, D. Transformaciones globales. (2002). Oxford University Press. México.

Kotler, M. (2000). Consecuencias de la globalización para el desarrollo industrial. Editorial Onudi. París.

Lall, S. (1992). Competitividad; Inversiones Extranjeras; Inversiones Directas; Tecnología; Empresas transnacionales. UNCTAD. 11(3). 23-35, diciembre.

Lavagna, R. (1999). Neo-conservadorismo versus capitalismo competitivo. Fondo de Cultura Económica. Buenos Aires.

Manzanares, M. A. (1996). La Formación en la Empresa ante la Innovación Tecnológica. Rev. Dirección y Organización. 24(16), 16-22. Abril. Madrid

Putnam, M. (1993). Las Pymes y su rol dentro de las variables económicas. Ed. Alianza. Madrid.

Rosenberg, D. y Rolt, J. (1989). Continuidad y discontinuidad en el cambio tecnológico. Sobre la importancia de la imitación, la actividad incremental y gradual de las empresas. Grupo Editorial. Madrid.

Ruggie, J. (1993). Territoriality and beyond. International Organization. 13(41). 115.

Schumpeter, J. A. (1994). Historia del análisis económico, Ariel, Barcelona.

Thurow, L. (1996) .El futuro del capitalismo. Como la economía de hoy determina el mundo del mañana. Editorial Javier Vergara. Buenos Aires.

\footnotetext{
(c) (1)(2)

La Revista Estudios es editada por la Universidad de Costa Rica y se distribuye bajo una Licencia Creative Commons Atribución-NoComercial-CompartirIgual 3.0 Costa Rica. Para más información envíe un mensaje a revistaestudios.eeg@ucr.ac.cr.
} 